\title{
PERSPECTIVE
}

\section{A Health Services Research Agenda for Bariatric Surgery Within the Veterans Health Administration}

\author{
L. M. Funk, MD, MPH ${ }^{1,2}$, W. Gunnar, MD, JD³, J. A. Dominitz, MD, MHS ${ }^{4,5}$, D. Eisenberg, MD, MS 6,7 , \\ S. Frayne, MD, MPH ${ }^{6,8}$, M. Maggard-Gibbons, MD, MSHS ${ }^{9,10}$, M. A. Kalarchian, $P h D^{11}$, \\ E. Livingston, $M D^{12,13}$, V. Sanchez, $M D^{14,15}$, B. R. Smith, MD ${ }^{16,17}$, H. Weidenbacher, PhD ${ }^{18,19}$, and \\ Matthew L. Maciejewski, PhD ${ }^{18,19}$
}

\begin{abstract}
${ }^{7}$ William S. Middleton VA Hospital, Madison, WI, USA; ${ }^{2}$ Department of Surgery, University of Wisconsin-Madison, Madison, WI, USA; ${ }^{3}$ The George Washington University, Washington, DC, USA; ${ }^{4}$ U.S. Department of Veterans Affairs, Washington, DC, USA; ${ }^{5}$ Division of Gastroenterology, University of Washington, Seattle, WA, USA; ${ }^{6}$ VA Palo Alto Health Care System, Palo Alto, CA, USA; ${ }^{7}$ Department of Surgery, Stanford University School of Medicine, Stanford, CA, USA; ${ }^{8}$ Department of Medicine, Stanford University School of Medicine, Stanford, CA, USA; ${ }^{9}$ VA Greater Los Angeles Healthcare System, Los Angeles, CA, USA; ${ }^{10}$ Department of Surgery, David Geffen School of Medicine at the University of California, Los Angeles, CA, USA; ${ }^{11}$ School of Nursing and Department of Psychology, Duquesne University, Pittsburgh, PA, USA; ${ }^{2}$ JAMA, Chicago, IL, USA; ${ }^{13}$ Department of Surgery at the UT Southwestern School of Medicine, Dallas, TX, USA; ${ }^{14}$ VA Boston Healthcare System, Boston, MA, USA; ${ }^{15}$ Boston University School of Medicine, Boston, MA, USA; ${ }^{16}$ VA Long Beach Healthcare System, Long Beach, CA, USA; ${ }^{17}$ Department of Surgery, UC Irvine Medical Center, Irvine, CA, USA; ${ }^{18}$ Center for Health Services Research in Primary Care (152), Durham VA Medical Center, Durham, NC, USA; ${ }^{19}$ Division of General Internal Medicine, Department of Medicine, Duke University, Durham, NC, USA.
\end{abstract}

In 2016, the Veterans Health Administration (VHA) held a Weight Management State of the Art conference to identify evidence gaps and develop a research agenda for population-based weight management for veterans. Included were behavioral, pharmacologic, and bariatric surgery workgroups. This article summarizes the bariatric surgery workgroup (BSWG) findings and recommendations for future research. The BSWG agreed that there is evidence from randomized trials and large observational studies suggesting that bariatric surgery is superior to medical therapy for short- and intermediate-term remission of type 2 diabetes, long-term weight loss, and long-term survival. Priority evidence gaps include long-term comorbidity remission, mental health, substance abuse, and health care costs. Evidence of the role of endoscopic weight loss options is also lacking. The BSWG also noted the limited evidence regarding optimal timing for bariatric surgery referral, barriers to bariatric surgery itself, and management of high-risk bariatric surgery patients. Clinical trials of pre- and postsurgery interventions may help to optimize patient outcomes. A registry of overweight and obese veterans and a workforce assessment to determine the VHA's capacity to increase bariatric surgery access were recommended. These will help inform policy modifications and focus the research agenda to improve the ability of the VHA to deliver population-based weight management.

KEY WORDS: bariatric surgery; Veterans Health Administration; health services research; weight loss; veterans.

JGenInternMed32(Supp11):S65-S9

DOI: $10.1007 / \mathrm{s} 11606-016-3951-4$

() Society of General Internal Medicine 2016

\section{INTRODUCTION}

Given the high prevalence of obesity among veterans, there is an urgent need to develop population-based weight management services. ${ }^{1}$ In 2006, the Veterans Health Administration

Published online March 7, 2017
(VHA) MOVE! program was established and disseminated to all Department of Veterans Affairs (VA) hospitals, offering behavioral weight management strategies for the 3.9 million veterans who were eligible (body mass index $[\mathrm{BMI}] \geq 30$, or $25-30$ and an obesity-related comorbidity). ${ }^{2,3}$ Of the 136,982 veterans who completed a MOVE! visit in 2015, more than 65,000 were severely obese (class II or III). ${ }^{4}$ In 2000 , more than 200,000 veterans met the BMI criteria for bariatric surgery established by the National Institutes of Health (NIH). ${ }^{5,6}$ All veterans who meet NIH criteria and bariatric programspecific criteria are eligible for VA-financed bariatric surgery. However, only a fraction, approximately 450 veterans with severe obesity, undergo bariatric surgery annually within the VA system. ${ }^{4}$

Bariatric surgery has changed dramatically over the past 25 years, from a strictly open surgical approach to a laparoscopic procedure. Laparoscopic vertical sleeve gastrectomy and Roux en-Y-gastric bypass are now the most common bariatric operations in the U.S. ${ }^{7}$ Short-term complication and mortality rates have dropped substantially. ${ }^{8,9}$ In 2013, a symposium convened by the $\mathrm{NIH}^{10}$ acknowledged substantial improvement in the safety and efficacy of bariatric surgery since the initial NIH consensus panel in $1991 .^{6}$ The 2013 symposium report highlighted the need for evidence on long-term outcomes such as survival, disease remission, and costs. ${ }^{10}$

Given the rapidly evolving literature regarding bariatric surgery and the need to identify evidence gaps in order to establish research priorities, the VA Health Services Research and Development Service (HSR\&D) sponsored a Weight Management State of the Art (SOTA) conference in March 2016. SOTAs bring together a multidisciplinary group of VA and non-VA experts to synthesize what is and is not known about topics critical to the health and well-being of veterans, and to promote implementation of findings that 
improve quality of care. Three workgroups (behavioral, pharmacologic, and bariatric surgery) were created. The goals of the Bariatric Surgery Workgroup (BSWG) were to characterize what is known about four key questions developed by the SOTA planning committee and workgroup co-leads (LF, MM) and to clarify policy and research priorities. This report summarizes the discussions and conclusions from the BSWG.

\section{METHODS}

Conference co-chairs and workgroup co-leads conferred with planning committee members to invite BSWG members to address key areas where there was sufficient evidence to inform VA policy or where significant evidence gaps existed. The BSWG consisted of the VHA National Director of Surgery (WG), five bariatric surgeons (DE, LF, EL, BS, VS), a general surgeon (MG), two psychologists (MK, HW), an internal medicine physician (SF), the VHA National Program Director for Gastroenterology (JD), and a health economist (MM).

A cyberseminar held on January 15, 2016, oriented participants to the SOTA objectives. BSWG members were asked to consider four key questions after reviewing several systematic reviews ${ }^{11-15}$ and peer-reviewed papers, ${ }^{10,16,17}$ and the group met face-to face in Washington, DC, on March 7, 2016. A recorder (HW) kept a written record of the discussion, which became the basis for a presentation to all SOTA participants on March 8, 2016, and also served as the basis for this report. During the March 7 discussion, BSWG members agreed that it was important to understand the variability among VHA bariatric surgery programs. This led the National Director of Surgery to survey the 21 VHA bariatric surgery programs during March-April 2016 regarding their pre-surgery requirements and their ability to increase their surgical volume. The survey questions were sent to BSWG members for feedback prior to being circulated to each bariatric program.

\section{RESULTS}

\section{Key Question \# 1: What Is the Average Effect of Bariatric Surgery on Short-Term and Long-Term Weight Loss, Comorbidity Resolution, Compli- cations, Mortality, and Costs?}

The BSWG considered evidence regarding patient outcomes according to time periods recommended by the American Society for Metabolic and Bariatric Surgery: short-term ( $<3$ years after surgery), medium-term $(\geq 3$ and $<5$ years), and long-term ( $\geq 5$ years). ${ }^{18}$ BSWG members identified important outcomes related to bariatric surgery including weight loss, change in relevant health conditions (type 2 diabetes, hypertension, cardiovascular disease, obstructive sleep apnea [OSA], gastroesophageal reflux disease [GERD], dyslipidemia, urinary incontinence, cancer, eating disorders, depression, post-traumatic stress disorder, schizophrenia, substance use disorders), quality of life, functional status, health care costs, postoperative complications and mortality.

There was consensus that the short-term evidence regarding the beneficial effect of bariatric surgery was present in systematic reviews, based on observational and randomized studies for postoperative weight loss, ${ }^{10,11,19}$ type 2 diabetes remission, ${ }^{10,11,19}$ hypertension remission, ${ }^{11,19}$ dyslipidemia remission, ${ }^{11,19}$ and improved survival. ${ }^{10,11,19}$ There was also consensus regarding the acceptable overall rate of short-term complications and low mortality associated with bariatric surgery. ${ }^{11,19}$ Evidence on medium-term weight loss, diabetes and dyslipidemia remission, and improved survival among bariatric surgery patients was also available from systematic reviews and observational studies. ${ }^{10,20}$ Long-term evidence regarding weight loss and improved survival associated with bariatric surgery was available primarily in large observational studies. ${ }^{16,21-23}$

The BSWG found mixed literature on health care costs following bariatric surgery, based mostly on non-veteran cohorts. ${ }^{24}$ Only one US-based cost analysis followed patients for at least 5 years. ${ }^{25}$ Evidence about mental health outcomes was also somewhat limited. A 2014 VA report and recent metaanalysis noted that bariatric surgery was associated with lower rates of depression and improved quality of life after surgery. ${ }^{26,27}$

Priority Areas for Research. Understanding the short- and long-term impact of bariatric surgery on mental health outcomes was a priority for workgroup members, as were studies examining the impact of bariatric surgery on long-term complications, GERD, OSA, substance abuse, and health care costs. BSWG members agreed that it was also important to examine heterogeneity in weight loss and associated outcomes according to patient characteristics, type of surgical procedure, and health system characteristics. Workgroup members recommended the creation of a VHA registry of overweight, obese, and severely obese veterans that would prospectively track patient outcomes throughout the VA.

\section{Key Question \#2: How Long Should Behavioral Interventions Be Pursued Before Considering Surgery? Is There a Definition of Success or Failure That Should Prompt Bariatric Surgery Referral?}

The BSWG discussed evidence regarding the association between pre-surgery dietary or weight loss requirements and post-surgery outcomes. There was no evidence that preoperative weight loss mandates established by payers improved postoperative outcomes. ${ }^{14}$ However, regimens delivered in the preoperative period, such as liquid diets, may be associated with greater weight loss after bariatric surgery and lower postoperative complication rates. ${ }^{13,28}$ 
BSWG members found it difficult to identify evidencebased definitions for "failure" that should prompt bariatric surgery referral. Patient engagement with the bariatric program was thought to be a key factor in identifying optimal surgical candidates. There was variation in how BSWG members defined engagement. For some, it meant adhering to preoperative weight loss requirements; for others, consistent attendance at pre-surgery visits represented engagement.

Priority Areas for Research. Developing metrics for measuring "success" or "failure" in response to behavioral and/or pharmacologic interventions that should prompt bariatric surgery referral was seen by the BSWG as important. Members thought that future research efforts should focus on integrating MOVE! with pharmacological treatment and surgery. Adoption of standardized metrics for measuring bariatric surgery outcomes was considered important.

Traditional randomized trials or newer adaptive designs could be used to examine whether structured pre- or postsurgery behavioral or pharmacological interventions could improve outcomes. Clarification of the factors indicative of sufficient patient engagement in the bariatric surgery screening and evaluation process was identified as a key need. There was consensus among the group that high patient engagement is critical for optimal outcomes. A patient engagement measure could be useful in characterizing patient and provider factors associated with optimal post-surgical outcomes and may be useful to bariatric surgery teams when determining patient eligibility.

All 21 bariatric surgery programs in the VA responded to the survey administered by the National Director of Surgery. Nearly all $(86 \%, n=18)$ programs required veterans to lose at least $5 \%$ body weight prior to surgery, and $70 \%(n=15)$ required MOVE! attendance as a condition of eligibility for surgery. Less than half $(43 \%, n=9)$ had fast-track programs in place for special populations (e.g., patients with BMI scores too high to qualify for joint replacements). If more candidates were referred for bariatric surgery, the VA would be capable of meeting some of this demand: $76 \%(n=16)$ programs reported that they could increase their bariatric surgical volume by $25 \%$ or more without additional resources.

\section{Key Question \#3: What Are the Patient-, Provider-, and System-Level Barriers to Bariatric Surgery Referral and Receipt?}

BSWG members agreed that there was limited literature regarding barriers to and facilitators of referral and uptake of bariatric surgery, as only one systematic review was identified (patient and referring provider knowledge about bariatric surgery were notable barriers). ${ }^{12}$ The BSWG identified the following as potential barriers: limited patient and provider knowledge, coordination of care across VA sites, a low number of VA facilities that perform bariatric surgery, limited surgical volume in those centers, and travel distances. Process mapping addressing barriers to bariatric surgery could support development of a standardized process to identify and refer candidates for bariatric surgery. At the initiation of the National Director of Surgery, the VA will administer a national survey to inform process mapping and support MOVE! refinement and other key referral sources for bariatric surgery.

Priority Areas for Research. There was workgroup consensus that four steps would help optimize bariatric surgery care throughout the VHA: 1) establishing service line agreements between departments of surgery, primary care, and others (e.g., nutrition, mental health) to facilitate care coordination; 2) initiating a patient registry as described above; 3 ) improving clinical and patient decision support; and 4) creating dashboards to track performance.

\section{Key Question \#4: What Strategies Should Be Considered Before Performing Bariatric Surgery in "Special Populations" of Bariatric Surgery Patients, Including the Super-Obese, High- Medical-Risk Patients, and Those Whose BMI is Too High for Other Types of Surgery (i.e., Ortho- pedic Surgery or Transplantation)? What is the Role of Endoscopic Interventions for Weight Loss (e.g., Intragastric Balloon)?}

The workgroup noted that there was no established protocol for managing veterans with high BMI, who are at higher risk for adverse events and are more technically challenging to operate on. The VHA Quality Enhancement Research Initiative (QUERI) assessed the comparative effectiveness of bariatric surgery for patients with BMI $\geq 50$ in preparation for the SOTA. ${ }^{15}$ There was limited evidence specifying the benefits and harms of this type of surgery for patients with a BMI $\geq 50$ compared to non-surgical treatment. It was agreed that there is insufficient literature on outcomes for super-obese bariatric surgery patients, primarily because they are typically combined with lower-BMI patients (BMI 35-50) in the analyses. The body of work on the effectiveness of bariatric surgery includes many patients with $\mathrm{BMI} \geq 50$.

Priority Areas for Research. Future research should examine whether outcomes differ for patients with a higher BMI cutoff, such as $>60$, which most bariatric surgeons in the workgroup felt was appropriate. Given the unique intraoperative technical and physiological challenges that these patients present, the benefits of atypical bariatric surgery treatment pathways such as a very-low-calorie diet (VLCD) before bariatric surgery should be examined.

One major recommendation from the group was that further research be conducted regarding outcomes 
following placement of intragastric balloons (IGBs), which have been approved by the Food and Drug Administration (FDA) for patients with a BMI of 30-40 and at least one obesity-related comorbidity. ${ }^{29}$ Study designs for randomized trials involving IGBs for three special populations were discussed: 1) patients with higher BMI (e.g., BMI $\geq 60$ ); 2) patients who are ineligible for other operations until they lose a significant amount of weight; and 3) high-medical-risk patients (e.g., severe congestive heart failure, oxygen dependence). Post-marketing surveillance of patient outcomes would be critical if IGB use were to increase among veteran populations.

\section{CONCLUSIONS}

A comprehensive research and clinical management plan for veterans with obesity will be crucial over the next several decades, and bariatric surgery will be an important part of this multidisciplinary effort. To inform this effort, the group identified and prioritized several research gaps, including developing effective preoperative and postoperative interventions, documenting long-term bariatric surgery outcomes, and establishing a registry of obese veterans. Results from the preliminary survey of bariatric surgery programs suggest that there is adequate infrastructure within the VA system to increase surgical volume in the short term. A comprehensive workforce assessment would help further elucidate the VHA's long-term capacity to increase access. A better understanding of the patient, provider, and health system barriers to and facilitators of bariatric surgery care was also perceived as critical. Special populations of patients with severe obesity may need tailored obesity-care pathways to optimize outcomes. Given veterans' longitudinal use of VHA care and a patient-accessible electronic health record, the VHA is ideally positioned to integrate behavioral, pharmacological, and surgical approaches to longterm management of obesity, a development that could improve patient outcomes and move the field forward.

Acknowledgments: The Weight Management SOTA was funded by the VA Health Services Research and Development Service. The committee presented its findings on day 2 of the Weight Management SOTA on March 8, 2016. The report outlined here was supported by the Department of Veterans Affairs, Veterans Health Administration, Health Services Research and Development Service. Dr. Funk is a VA HSR\&D Career Development awardee at the William S. Middleton VA in Madison, WI. Dr. Maciejewski received support from the VA HSR\&D for a Research Career Scientist award (RCS 10-391). All commentary and conclusions expressed in this article are those of the authors, and do not represent the opinions of the Health Services Research and Development Service or the Department of Veterans Affairs.

Corresponding Author: Matthew L. Maciejewski, PhD; Center for Health Services Research in Primary Care (152)Durham VA Medical Center, 411 West Chapel Hill Street, Suite 600, Durham, NC 27705, USA (e-mail: matthew.maciejewski@va.gov).

Compliance with Ethical Standards:

Conflict of Interest: The authors declare that they have no conflict of interest.

\section{REFERENCES}

1. Rush T, LeardMann CA, Crum-Cianflone NF. Obesity and associated adverse health outcomes among US military members and veterans: findings from the millennium cohort study. Obesity (Silver Spring). 2016;24:1582-9.

2. Kahwati LC, Lance TX, Jones KR, Kinsinger LS. RE-AIM evaluation of the Veterans Health Administration's MOVE! Weight Management Program. Transl Behav Med. 2011;1:551-60.

3. Kinsinger LS, Jones KR, Kahwati L, Harvey R, Burdick M, Zele V, et al. Design and dissemination of the MOVE! Weight-management program for veterans. Prev Chronic Dis. 2009;6:A98.

4. Communication with the VHA Director of Surgery. 2016.

5. Das SR, Kinsinger LS, Yancy WS Jr, Wang A, Ciesco E, Burdick M, et al. Obesity prevalence among veterans at Veterans Affairs medical facilities. Am J Prev Med. 2005;28:291-4.

6. Gastrointestinal surgery for severe obesity. NIH consensus development conference, March 25-7, 1991. Nutrition. 1996;12:397-404.

7. MBSAQIP Semiannual Report: American College of Surgeons, American Society for Metabolic and Bariatric Surgery; June 2014.

8. Flum DR, Salem L, Elrod JA, Dellinger EP, Cheadle A, Chan L. Early mortality among medicare beneficiaries undergoing bariatric surgical procedures. JAMA. 2005;294:1903-8.

9. Longitudinal Assessment of Bariatric Surgery C, Flum DR, Belle SH, King WC, Wahed AS, Berk $\mathbf{P}$, et al. Perioperative safety in the longitudinal assessment of bariatric surgery. N Engl J Med. 2009;361:445-54.

10. Courcoulas AP, Yanovski SZ, Bonds D, Eggerman TL, Horlick M, Staten MA, et al. Long-term outcomes of bariatric surgery: a National Institutes of Health symposium. JAMA Surg. 2014;149:1323-9.

11. Chang SH, Stoll CR, Song J, Varela JE, Eagon CJ, Colditz GA. The effectiveness and risks of bariatric surgery: an updated systematic review and meta-analysis, 2003-2012. JAMA Surg. 2013;149:275-87.

12. Funk LM, Jolles S, Fischer LE, Voils CI. Patient and referring practitioner characteristics associated with the likelihood of undergoing bariatric surgery: a systematic review. JAMA Surg. 2015;150:999-1005.

13. Gerber P, Anderin C, Thorell A. Weight loss prior to bariatric surgery: an updated review of the literature. Scand J Surg. 2015;104:33-9.

14. Ochner CN, Dambkowski CL, Yeomans BL, Teixeira J, Xavier PiSunyer F. Pre-bariatric surgery weight loss requirements and the effect of preoperative weight loss on postoperative outcome. Int $\mathrm{J}$ Obes (Lond). 2012;36:1380-7.

15. Peterson K, Anderson J, Ferguson L, Erickson K, Hymphrey L. Evidence brief: comparative effectiveness of bariatric surgery in super obesity (BMI > $50 \mathrm{~kg} / \mathrm{m} 2$ ). VA ESP Project \#09-199 2015.

16. Arterburn DE, Olsen MK, Smith VA, Livingston EH, Van Scoyoc L, Yancy WS Jr, et al. Association between bariatric surgery and long-term survival. JAMA. 2015;313:62-70.

17. Finkelstein EA, Allaire BT, Globe D, Dixon JB. The business case for bariatric surgery revisited: a non-randomized case-control study. PLoS One. 2013;8:e75498.

18. Brethauer SA, Kim J, El Chaar M, Papasavas P, Eisenberg D, Rogers A, et al. Standardized outcomes reporting in metabolic and bariatric surgery. Obes Surg. 2015;25:587-606.

19. Puzziferri N, Roshek TB 3rd, Mayo HG, Gallagher R, Belle SH, Livingston EH. Long-term follow-up after bariatric surgery: a systematic review. JAMA. 2014;312:934-42.

20. Arterburn DE, Courcoulas AP. Bariatric surgery for obesity and metabolic conditions in adults. BMJ. 2014;349:g3961.

21. Sjostrom L, Lindroos AK, Peltonen M, Torgerson J, Bouchard C, Carlsson B, et al. Lifestyle, diabetes, and cardiovascular risk factors 10 years after bariatric surgery. N Engl J Med. 2004;351:2683-93.

22. Adams TD, Gress RE, Smith SC, Halverson RC, Simper sC, Rosamond WD, et al. Long-term mortality after gastric bypass surgery. N Engl J Med. 2007;357:753-61.

23. Maciejewski ML, Arterburn DE, L.V. VS, Smith VA, Yancy WS, Weidenbacher $\mathbf{H}$, et al. Bariatric surgery and long-term durability of weight loss. JAMA Surg. 2016;151(11):1046-55.

24. Maciejewski ML, Arterburn DE., L. Van Scoyoc, V.A. Smith, W.S. Yancy, H. Weidenbacher, E . .H. Livingston, M.K. Olsen 2016. "Bariatric Surgery and Long-Term Durability of Weight Loss”, JAMA Surgery, 151(11): 1046-1055.

25. Weiner JP, Goodwin SM, Chang HY, Bolen SD, Richards TM, Johns RA, et al. Impact of bariatric surgery on health care costs of obese persons: a 6-year follow-up of surgical and comparison cohorts using health plan data. JAMA Surg. 2013;148:555-62. 
26. Gibbons MM, Maher AR, Dawes AJ, Booth MS, Miake-Lye IM, Beroes JM, et al. Mental health assessment and psychosocial interventions for bariatric surgery. VA evidence-based synthesis program reports. Washington, DC; 2014.

27. Dawes AJ, Maggard-Gibbons M, Maher AR, Booth MJ, Miake-Lye I, Beroes JM, et al. Mental health conditions among patients seeking and undergoing bariatric surgery: a meta-analysis. JAMA. 2016;315:150-63.
28. Livhits M, Mercado C, Yermilov I, Parikh JA, Dutson E, Mehran A, et al. Does weight loss immediately before bariatric surgery improve outcomes: a systematic review. Surg Obes Relat Dis. 2009;5:713-21.

29. FDA approves non-surgical temporary balloon device to treat obesity. (Accessed May 25, 2016, at http://www.fda.gov/NewsEvents/Newsroom/PressAnnouncements/ucm456296.htm.) 